\title{
95. Remarks on a Theorem concerning Conformal Transformations
}

\author{
By Yoshihiro TASHIRo \\ Department of Mathematics, Okayama University, Okayama, Japan
}

(Comm. by Z. SuetunA, M.J.A., Oct. 12, 1959)

In a recent paper $\mathrm{K}$. Yano and T. Nagano [3] proved the following

Theorem A. Let $M$ be a complete Einstein manifold and suppose that a vector field on $M$ generates globally a one-parameter group of non-homothetic conformal transformations. ${ }^{1}$ Then $M$ is isometric to a spherical space, i.e. a simply connected, complete space of positive constant sectional curvature. In particular $M$ is homeomorphic to the sphere $S^{n}$.

On the other hand, S. Ishihara and the present author [1] investigated the topological and differential-geometrical properties of compact or complete Riemannian manifolds admitting a concircular transformation. A concircular transformation of a Riemannian manifold $M$ with metric $g_{\mu \lambda}$ into a Riemannian manifold ' $M$ with metric ' $g_{\mu \lambda}$ is by definition a conformal transformation

$$
{ }^{\prime} g_{\mu \lambda}=\rho^{2} g_{\mu \lambda},
$$

which carries geodesic circles in $M$ to geodesic circles in ' $M$, and is characterized by the equation

$$
\nabla_{\mu} \rho_{\lambda}-\rho_{\mu} \rho_{\lambda}=\psi g_{\mu \lambda},
$$

where $\rho$ is a positive-valued function on $M, \rho_{\lambda}=\nabla_{\lambda} \log \rho$ and $\psi$ is a function on $M$. We obtained the following theorems:

Theorem B. Let $M$ and ' $M$ be Riemannian manifolds whose scalar curvatures $k$ and ' $k$ are constant. We assume that $M$ is complete and that there exists a concircular transformation of $M$ into 'M. Then the manifold $M$ is

I) a Euclidean space, if $k=0$,

II) a spherical space, if $k>0$, or

III) a hyperbolic space, if $k<0$. $^{2)}$

Theorem C. In addition to the assumptions of Theorem $\mathrm{B}$, assume that ' $M$ is complete too and the concircular transformation

1) In this paper we suppose that manifolds are always connected, of dimension $n>2$ and of class $C^{\infty}$, and that the differentiability of transformations and quantities is also of class $C^{\infty}$. Greek indices run from 1 to $n$. We shall deal only with nonhomothetic conformal transformations, and the term "conformal" will always mean "non-homothetic conformal".

2) The scalar curvatures in this paper are different from those in [7] in the sign. 
is a homeomorphism of $M$ onto ' $M$. Then the scalar curvatures $k$ and ' $k$ should be positive and both $M$ and 'M are spherical spaces.

First we notice the following

Theorem 1. In order that a conformal transformation map an Einstein manifold into an Einstein one, it is necessary and sufficient that the transformation be concircular.

Proof. The sufficiency was given by K. Yano [2]. For a conformal transformation (1), it is well known that we have

$$
\begin{aligned}
& { }^{\prime} K_{\nu \mu \lambda}{ }^{k}=K_{\nu \mu \lambda}{ }^{k}-A_{\nu}{ }^{\kappa} \rho_{\mu \lambda}+A_{\mu}{ }^{k} \rho_{\nu \lambda}-\rho_{\nu}{ }^{k} g_{\mu \lambda}+{ }{ }^{k}{ }^{k} g_{\nu \lambda} \text {, } \\
& { }^{\prime} K_{\mu \lambda}=K_{\mu \lambda}-(n-2) \rho_{\mu \lambda}-g_{\mu \lambda} \rho_{k}{ }^{k} \text {, } \\
& n^{\prime} k \rho^{2}=n k-2 \rho_{\kappa}{ }^{k} \text {, }
\end{aligned}
$$

where $K_{\nu \mu \lambda}{ }^{k}, K_{\mu \lambda}$ and $k$ are the curvature tensor, the Ricci tensor and the scalar curvature of $M$ respectively, the prime indicates the corresponding quantities of ' $M$ and we have put

$$
\rho_{\mu \lambda}=\nabla_{\mu} \rho_{\lambda}-\rho_{\mu} \rho_{\lambda}+\frac{1}{2} g_{\mu \lambda} \rho_{\kappa} \rho^{\kappa} \text {. }
$$

If $M$ and ' $M$ are Einstein manifolds,

$$
\begin{aligned}
& K_{\mu \lambda}=(n-1) k g_{\mu \lambda}, \\
& { }^{\prime} K_{\mu \lambda}=(n-1)^{\prime} k^{\prime} g_{\mu \lambda}=(n-1)^{\prime} k \rho^{2} g_{\mu \lambda},
\end{aligned}
$$

then, from (4) and (5), we have

$$
\rho_{\mu \lambda}=\frac{1}{2}\left(k-{ }^{\prime} k \rho^{2}\right) g_{\mu \lambda}
$$

or

$$
\nabla_{\mu} \rho_{\lambda}-\rho_{\mu} \rho_{\lambda}=\frac{1}{2}\left(k-{ }^{\prime} k \rho^{2}-\rho_{\kappa} \rho^{k}\right) g_{\mu \lambda} .
$$

Hence the conformal transformation is concircular.

Combining Theorems $\mathrm{B}$ and $\mathrm{C}$ with this theorem, we can generalize the Yano and Nagano's theorem as follows:

Theorem 2. If a complete Einstein manifold $M$ is transformed conformally into an Einstein one ' $M$, then the manifold $M$ is

I) a Euclidean space, if $k=0$,

II) a spherical space, if $k>0$, or

III) a hyperbolic space, if $k<0$.

Theorem 3. If a complete Einstein manifold $M$ admits a conformal transformation onto itself, then the manifold $M$ is a spherical space.

\section{References}

[1] S. Ishihara and Y. Tashiro: On Riemannian manifolds admitting a concircular transformation, Math. J. Okayama Univ., 9, 19-47 (1959).

[2] K. Yano: Concircular geometry, I-V, Proc. Imp. Acad., 16, 195-200, 354-360, 442-448, 505-511 (1940); 18, 446-451 (1942).

[3] K. Yano and T. Nagano: Einstein spaces admitting a one-parameter group of conformal transformations, Ann. Math., 69, 451-461 (1959). 